Virginia Commonwealth University VCU Scholars Compass

2008

\title{
Li- and B-decorated cis-polyacetylene: A computational study
}

S. Li

Virginia Commonwealth University

Puru Jena

Virginia Commonwealth University, pjena@vcu.edu

Follow this and additional works at: http://scholarscompass.vcu.edu/phys_pubs

Part of the Physics Commons

Li, S., Jena, P. Li- and B-decorated cis-polyacetylene: A computational study. Physical Review B, 77, 193101 (2008).

Copyright (C) 2008 American Physical Society.

\section{Downloaded from}

http://scholarscompass.vcu.edu/phys_pubs/72

This Article is brought to you for free and open access by the Dept. of Physics at VCU Scholars Compass. It has been accepted for inclusion in Physics Publications by an authorized administrator of VCU Scholars Compass. For more information, please contact libcompass@vcu.edu. 


\title{
Li- and B-decorated cis-polyacetylene: A computational study
}

\author{
S. Li and P. Jena \\ Physics Department, Virginia Commonwealth University, Richmond, Virginia 23284-2000, USA \\ (Received 23 August 2007; revised manuscript received 25 March 2008; published 5 May 2008)
}

\begin{abstract}
By using density functional theory and the generalized gradient approximation, we show that Li-decorated cis-polyacetylene meets some of the requirements of an ideal hydrogen storage material. Unlike Ti-doped cis-polyacetylene, Li resists clustering and can reversibly store up to $10.8 \mathrm{wt} \%$ hydrogen in molecular form. However, molecular dynamics simulations show that Li can retain hydrogen only at cryogenic temperatures. On the other hand, B-doped cis-polyacetylene can store up to $7.5 \mathrm{wt} \%$ hydrogen, but it binds to hydrogen too strongly to be suitable for room temperature applications. The results are compared to those in Ti-doped cis-polyacetylene.
\end{abstract}

DOI: 10.1103/PhysRevB.77.193101

PACS number(s): 71.15.Nc, 68.43.Bc, 84.60.Ve

The growing demand for energy, which is fueled by increasing population and rising standard of living in the developing world, has made it imperative that new sources of energy be found to sustain the global economy. The dwindling supply of fossil fuels and its adverse effect on global warming and pollution further requires that the new energy sources ideally should be abundant, renewable, and pollution-free. The current interest in hydrogen as an alternative energy resource is because it is the third most abundant element on earth, is renewable, and can produce energy pollution-free. While considerable problems exist in realizing this reality, suitable means of storing hydrogen for the mobile industry, which consumes $75 \%$ of the current fossil fuels, is the most difficult challenge faced by the industry. The difficulty lies in the fact that there are no solid state materials that can store and release hydrogen with large gravimetric and volumetric densities at near ambient conditions. Complex light metal hydrides ${ }^{1}$ as well as carbon based materials such as nanotubes, ${ }^{2-4}$ fullerenes, ${ }^{5}$ and metal-organic-frameworks ${ }^{6}$ can store hydrogen, but they all have poor thermodynamics and kinetics. In the former, hydrogen binding is strong, while in the later, it is very weak. ${ }^{7,8}$ Recently, several attempts have been made to dope carbonbased materials with suitable metal atoms that can molecularly bind hydrogen and with a characteristic binding energy that is intermediate between physisorption and chemisorption. ${ }^{9-12}$ However, the stability of these materials during repeated hydrogen cycling has been an issue and no materials that are ideal for hydrogen storage have surfaced.

Recently, some authors have suggested that Ti-coated polymers, such as polyacetylene ${ }^{13}$ (PA) and ethylene complexes ${ }^{14}$ may be ideal for hydrogen storage as the gravimetric density in these materials can reach 7.6 and 14 wt \%, respectively. However, we have recently demonstrated that Ti atoms doped into PA will cluster and severely undermine its hydrogen storage capacity. ${ }^{15}$ Similar results have also been found for Ti-decorated ethylene complexes. Since both PA and ethylene are readily available materials, it would be ideal if other metal atoms could be found that will not only resist clustering but also absorb large amounts of hydrogen when doped with these metal atoms.

In this Brief Report, we present results on $\mathrm{Li}$ and $\mathrm{B}$ decorated cis-polyacetylene since both these elements have certain advantages. $\mathrm{Li}$ is not only a light element but also has a very low cohesive energy. Consequently, $\mathrm{Li}$ atoms on PA may resist clustering as they have been found in Li decorated fullerenes. Second, the charge transfer from $\mathrm{Li}$ atoms to the polymer, which is made possible due its low ionization potential, would allow the atoms to remain in a positively charge state. Through a charge polarization mechanism, the $\mathrm{Li}$ atoms can then bind to hydrogen molecules in molecular form. Li doping in graphene, ${ }^{16}$ fullerene, ${ }^{11}$ and carbon nanotubes ${ }^{17,18}$ has been reported to effectively improve the hydrogen uptake. Third, the synthesis of Li-decorated cis-PA in bulk quantities may not require an elaborate and expensive process. Boron like lithium is also a light element, but its chemistry is very different. For example, boron is a semimetal and forms two electron-three center bonds and has a complicated lattice structure. On the other hand, titanium is a transition metal element with large ionization potential and its properties are dominated by $3 d$ electrons, while the electronic structure of $\mathrm{Li}$ and $\mathrm{B}$ are governed by the $s$ and $p$ electrons.

To assess the potential of $\mathrm{Li}$ and B decorated PA for hydrogen storage, we have carried out self-consistent calculations of the total energy, equilibrium structure, and interaction of hydrogen molecules with Li- and B-doped cis-PAs. The results are compared to Ti-doped cis-PA. Spin-polarized calculations were carried out by using generalized gradient approximation ${ }^{19}$ to the density functional theory ${ }^{20}$ and the projector augmented wave ${ }^{21}$ (PAW) method. The PAW potentials with the valence states $2 s$ and $2 p$ for $\mathrm{Li}$ and $\mathrm{B}$ were used as prescribed in the Vienna ab initio simulation package (VASP). ${ }^{22}$ High precision calculations with a cutoff energy of $500 \mathrm{eV}$ for the plane-wave basis were performed. The ionic coordinates were optimized without any symmetry constraint. For the sampling of the irreducible wedge of the Brillouin zone, we used $k$-point grids of $8 \times 1 \times 1$ for the geometry optimization. Cohesive energies were calculated at the equilibrium volume. In all of the calculations, selfconsistency was achieved with a tolerance in the total energy of $0.1 \mathrm{meV}$. Hellman-Feynman force components on each ion in the supercells are converged to $5 \mathrm{meV} / \AA$. To determine the probability of clustering of $\mathrm{Li}$ and $\mathrm{B}$ on cis-PA, we compared the energy of two different structures, as shown in Figs. 1(a) and 1(b). In Fig. 1(a), note that the doped Li and B atoms are isolated from each other, while in Fig. 1(b), they form a chain. After determining the preferred site, we calcu- 


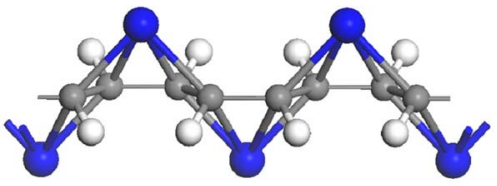

(a)

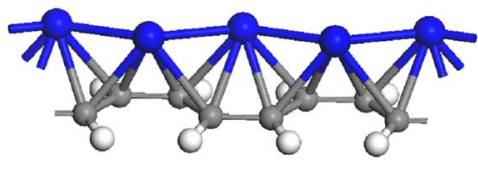

(b)
FIG. 1. (Color online) (a) Metal-decorated cis-PA where the dopant atoms do not cluster. (b) Metal-decorated cis-PA where the dopant atoms cluster. In both (a) and (b), two unit cells are shown. lated the maximum amount of stored hydrogen and their binding energies. In the following, we discuss our results.

We first optimized the equilibrium structure of $\mathrm{Li}$ decorated PA structure given in Fig. 1(a). Each Li is found to bind to four $\mathrm{C}$ atoms at two distances, i.e., 2.11 and $2.27 \AA$. Our calculated binding energy is $1.21 \mathrm{eV} / \mathrm{Li}$. To explore the possibility that Li atoms may cluster, we placed $\mathrm{Li}$ atoms on the same side of the chain [Fig. 1(b)]. The corresponding binding energy in Fig. 1(b) is found to be $1.19 \mathrm{eV} / \mathrm{Li}$. The $\mathrm{Li}$ atom is bound to four $\mathrm{C}$ atoms at two distances, 2.23 and $2.31 \AA$, and the Li-Li distance is $2.64 \AA$. This is in contrast to the Ti-doped cis-polyactylene, wherein the $\mathrm{Ti}$ atoms confined to the structure in Fig. 1(a) lie $0.85 \mathrm{eV}$ higher in energy than when allowed to cluster [Fig. 1(b)]. Thus, unlike Ti, Li atoms do not show a clear preference to cluster.

The electronic structures of cis-PA and Li-doped cis-PA are studied by calculating the respective densities of states. The results are shown in Fig. 2. The intrinsic cis-PA is a semiconductor with a band gap of $1.12 \mathrm{eV}$, which is compa-

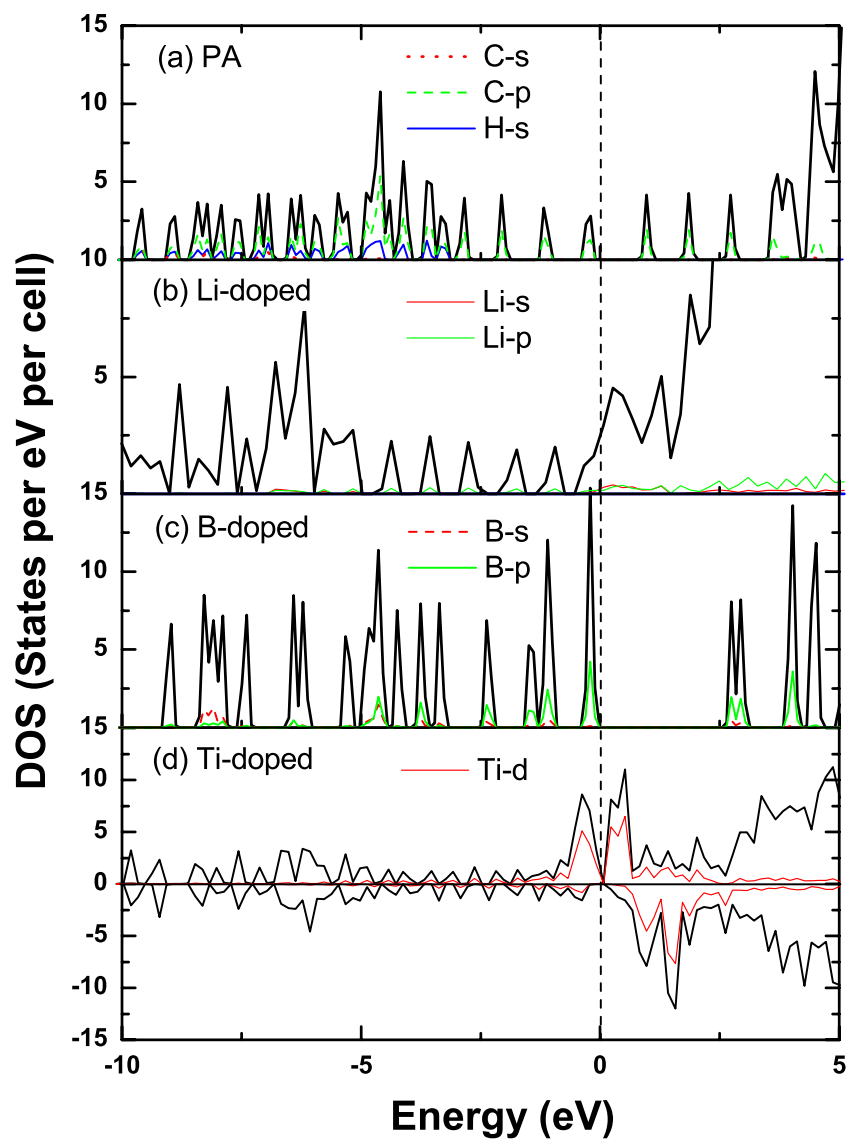

FIG. 2. (Color online) Calculated density of states for intrinsic, Li-doped, B-doped, and Ti-doped cis-PAs; the black solid lines represent the total density of states; Fermi level is set at zero. rable to $1.52 \mathrm{eV}$ reported for trans-PA. ${ }^{23}$ The lower valence band is composed of $\mathrm{C} s$ states, while the upper valence band is dominated by $\mathrm{C} p$ and $\mathrm{H} s$ states. When $\mathrm{Li}$ is doped, a nonmetal to metal transition takes place. Only a small portion of Li s state can be seen in the valence band and the coupling between $\mathrm{Li} s$ and $\mathrm{C} p$ states is not strong. For B-doped system, the situation is very different. Here, the band gap increases to $2.75 \mathrm{eV}$ with a strong coupling between B $p$ and C $p$ states in the valence band. Ti-doped PA presents an intermediate behavior where a pseudogap appears in the spin-up states. Unlike both Li- and B-doped PA, which are nonmagnetic, Ti-doped PA is magnetic. The magnetic moment on each $\mathrm{Ti}$ atom is $1.27 \mu_{B}$. The hybridization between Ti $d$ and $\mathrm{C} p$ states is strong. The hybridization between valence states of dopant and PA leads to different binding energies. For example, the binding energy of $\mathrm{Li}$ to $\mathrm{PA}$ is $1.21 \mathrm{eV} / \mathrm{Li}$ compared to $4.17 \mathrm{eV} / \mathrm{B}$ for boron and 3.29 $\mathrm{eV} / \mathrm{Ti}$ for titanium.

Next, we studied the energetics of hydrogen uptake by successively adding $\mathrm{H}_{2}$ molecules to the $\mathrm{Li}$ atoms and optimizing the resulting geometry. The results are shown in Fig. 3 . Note that the hydrogen atoms molecularly bind with a $\mathrm{H}-\mathrm{H}$ bond length of $0.76 \AA$. We were able to add up to three $\mathrm{H}_{2}$ molecules per $\mathrm{Li}$ atom. The energy gain in adding 1, 2, and $3 \mathrm{H}_{2}$ molecules to a $\mathrm{Li}$ atom are 0.1, 0.06, and

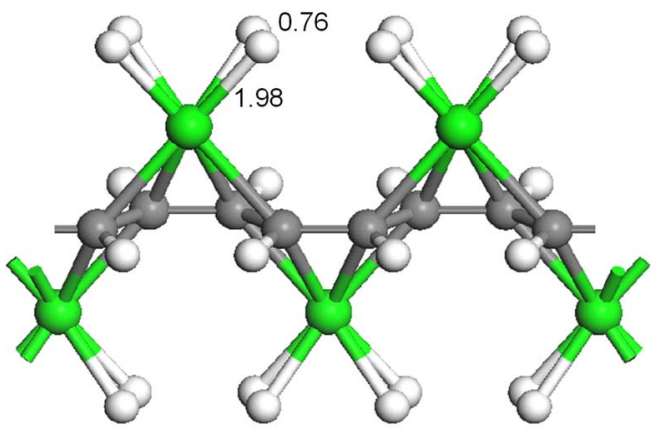

(a)

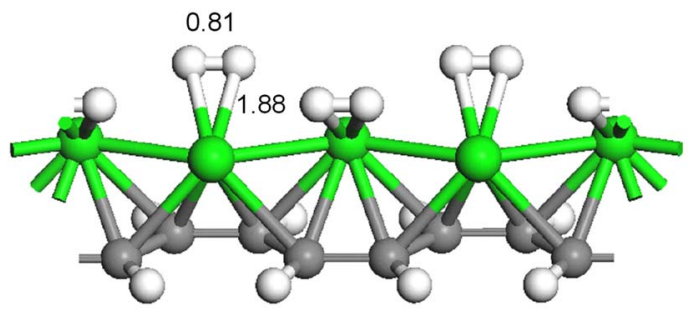

(b)

FIG. 3. (Color online) (a) Geometries of two $\mathrm{H}_{2}$ molecules bound to each isolated $\mathrm{Li}$ in $\mathrm{Li}_{2} \mathrm{C}_{4} \mathrm{H}_{4}$. (b) Geometries when one $\mathrm{H}_{2}$ molecule is bound to each $\mathrm{Li}$ in $\mathrm{Li}_{2} \mathrm{C}_{4} \mathrm{H}_{4}$ (clustered configuration). 
$0.03 \mathrm{eV} / \mathrm{H}_{2}$, respectively. When two $\mathrm{H}_{2}$ molecules are added to $\mathrm{Li}$, each $\mathrm{Li}$ atom is bound to $\mathrm{H}_{2}$ molecules at a distance of $1.98 \AA$ [see Fig. 3(a)]. The Li-C distances are slightly elongated from 2.11 to $2.12 \AA$ and from 2.27 to $2.33 \mathrm{~A}$. When the third $\mathrm{H}_{2}$ molecule is added, each $\mathrm{Li}$ is found to bind to these $\mathrm{H}_{2}$ molecules at distances of 2.04, 2.26, and $3.2 \AA$. The long bond over $3 \AA$ suggests that the third $\mathrm{H}_{2}$ molecule may not be useful as far as hydrogen storage is concerned. With two $\mathrm{H}_{2}$ molecules bound to each $\mathrm{Li}$ atom with an average binding energy of $0.08 \mathrm{eV} / \mathrm{H}_{2}$, the gravimetric density of Li-decorated cis-PA becomes 10.8 wt $\%$.

It is known that the $\mathrm{Li}^{+}$cation can bind up to six pairs of hydrogen molecules. In the present case, each Li can only adsorb two pairs of hydrogen molecules, and the average binding energy computed in the above is significantly smaller than $\mathrm{H}_{2}$ molecule bound to an isolated $\mathrm{Li}^{+}$ion $\left(0.25 \mathrm{eV} / \mathrm{H}_{2}\right){ }^{24}$ This suggests that the charge on the $\mathrm{Li}$ atom bound to the polymer is less than +1 . Even though the accuracy of atomic charge on Li computed from VASP may not be quantitative as it depends on the radius of muffin-tin sphere, the charge difference for the same type of atom with the same muffin-tin radius can still be used for qualitative analysis. Before hydrogen adsorption, the charge on $\mathrm{Li}$ is found to be +0.76 , and it remains nearly the same $(+0.74)$ when two $\mathrm{H}_{2}$ molecules are adsorbed.

We also considered the case that Li may cluster since the energy difference between clustering and isolated cases is very small. In this case, the maximum number of hydrogen molecules each Li can bind is one [Fig. 3(b)] with a binding energy of $0.1 \mathrm{eV}$. This leads to a gravimetric density of $5.7 \mathrm{wt} \%$. The $\mathrm{H}_{2}$ molecular bond is slightly elongated from 0.76 to $0.81 \AA$. The corresponding $\mathrm{Li}-\mathrm{H}$ distance is $1.88 \AA$.

Since the average binding energy of $\mathrm{H}_{2}$ to $\mathrm{Li}$ is small compared to that encountered in transition metal doped systems (here, the binding energies are between 0.5 to $1.0 \mathrm{eV} / \mathrm{H}_{2}$ ), we have carried out molecular dynamics (MD) simulations to study the temperature where hydrogen can be desorbed from Li-decorated cis-PA. We performed MD simulation at $77,150,200$, and $300 \mathrm{~K}$. For the first two temperatures, we used 10000 time steps, while for the later two, we used 5000, and 2000 time steps, respectively. Each step is $1 \mathrm{fs}$ long. The velocities were scaled at each time step. Each $\mathrm{Li}$ atom was found to be capable of binding to $2 \mathrm{H}_{2}$ molecules with a $\mathrm{Li}-\mathrm{H}$ distance of $1.98 \AA$ up to $10 \mathrm{ps}$ of MD simulations at $77 \mathrm{~K}$. Li can bind to only one $\mathrm{H}_{2}$ molecule at the temperature of $150 \mathrm{~K}$ up to $10 \mathrm{ps}$ simulation time. At 200 and $300 \mathrm{~K}$, both $\mathrm{H}_{2}$ molecules were found to move away after 5 and 2 ps, respectively. Thus, the Li coated cis-PA is able to store hydrogen only at moderately low temperatures.

Since the Li-H bond is very weak, we studied the possibility that the doping of $\mathrm{B}$ may prove to be advantageous without sacrificing the gravimetric density. B doping in single wall nanotube was found to improve the hydrogen uptake significantly, i.e., from $0.7 \%$ to $2.2 \%$ at room temperature. ${ }^{25}$ However, $\mathrm{B}$, like $\mathrm{Ti}$, is found to cluster. The configuration corresponding to Fig. 1(b) is $1.92 \mathrm{eV} / \mathrm{B}$ lower than the configuration corresponding to Fig. 1(a). The B atom was found to bind to the $\mathrm{C}_{4} \mathrm{H}_{4}$ chain with the shortest B-C distance of $1.66 \AA$ and the B-B distance of $1.51 \AA$.

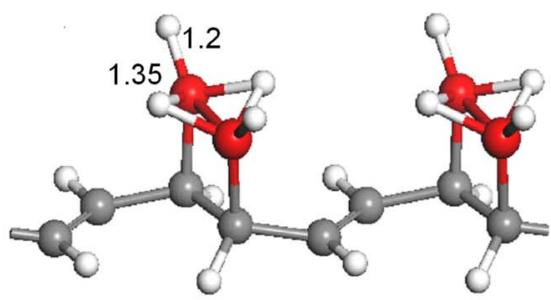

(a)

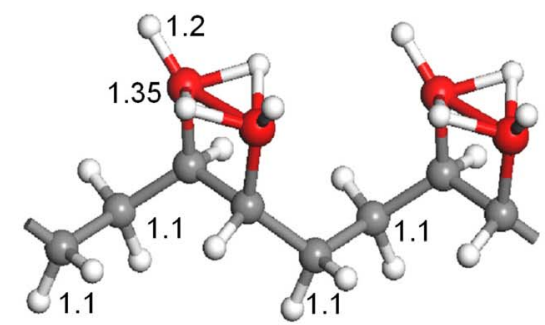

(b)

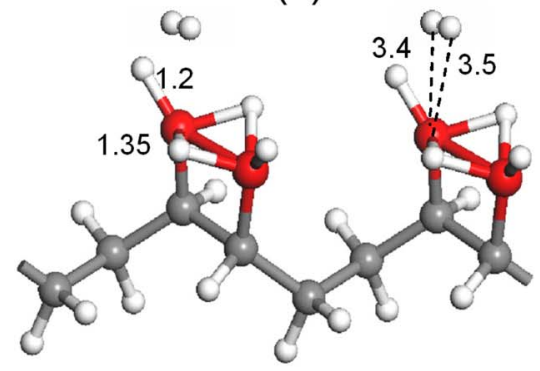

(c)

FIG. 4. (Color online) Geometries of $2 \mathrm{H}_{2}$ and $3 \mathrm{H}_{2}$, and $4 \mathrm{H}_{2}$ molecules bound to $\mathrm{B}_{2} \mathrm{C}_{4} \mathrm{H}_{4}$ (two unit cells are shown).

Unlike the Ti-doped cis-PA, the polymer chain is largely distorted and B atoms are not uniformly distributed. Instead, the $\mathrm{B}$ atoms form pairs and are separated from each other by a distance of $3.37 \AA$. The extremely high binding energy, 4.17 $\mathrm{eV} / \mathrm{B}$, shows that the B coated PA chain is very stable.

We next successively added hydrogen molecules to the $\mathrm{B}$-coated cis-PA chain. The energy gains in adding two, three, and four $\mathrm{H}_{2}$ molecules to two $\mathrm{B}$ atoms are 3.74, 1.38, and $0.02 \mathrm{eV}$, respectively. The first $\mathrm{H}_{2}$ molecule dissociates when added to a B atom and two $\mathrm{B}$ atoms bind to four $\mathrm{H}$ atoms with $\mathrm{B}-\mathrm{H}$ distances ranging between 1.2 and $1.35 \AA$. The corresponding B-B distance and the shortest B-C distance are 1.75 and $1.61 \AA$, respectively [Fig. 4(a)]. Note that hydrogen is chemisorbed and the B-H bond is much stronger than the $\mathrm{Li}-\mathrm{H}$ bond. When three hydrogen molecules were added to both $\mathrm{B}$ atoms, each $\mathrm{B}$ atom was found to dissociatively bind to one $\mathrm{H}_{2}$ molecule, while the third pair dissociated and bound to two $\mathrm{C}$ atoms on the chain [Fig. 4(b)]. This configuration yields a hydrogen gravimetric density of 7.5 wt \%. Addition of more $\mathrm{H}_{2}$ molecules is unfavorable since the fourth pair of $\mathrm{H}_{2}$ molecule is physisorbed with $\mathrm{B}-\mathrm{H}$ distance of $\sim 3.4 \AA$ [Fig. 4(c)]. Even though each B atom can bind two hydrogen atoms, the $\mathrm{H}$ atoms are chemisorbed and hard to desorb. Thus, we conclude that B-doped cis-PA may not be suitable as a hydrogen storage material. 
TABLE I. Energy differences $\Delta E\left(E_{(1 b)}-E_{(1 a)}\right)(\mathrm{eV})$ between clustered [Fig. 1(b)] and isolated configuration [Fig. 1(a)] of Ti, Li, and B coated cis-PAs. $D_{\mathrm{M}-\mathrm{H}}(\AA)$ and $E_{\mathrm{M}-\mathrm{H}}\left(\mathrm{eV} / \mathrm{H}_{2}\right)$ are, respectively, the distances between the dopant atom and hydrogen and the binding energy per $\mathrm{H}_{2}$. The positive entry in the first row indicates that dopants will not cluster, while negative entries suggest that dopants will cluster.

\begin{tabular}{lccc}
\hline \hline & $\mathrm{Ti}$ & $\mathrm{Li}$ & $\mathrm{B}$ \\
\hline$\Delta E\left(E_{(1 b)}-E_{(1 a)}\right)$ & -0.85 & 0.02 & -1.92 \\
$D_{\mathrm{M}-\mathrm{H}}$ & $1.85-2.05$ & 1.98 & $1.2-1.35$ \\
$E_{\mathrm{M}-\mathrm{H}}$ & 0.96 & 0.08 & 1.71 \\
wt $\%$ & 5.1 & 10.8 & 7.5 \\
\hline \hline
\end{tabular}

Our results are summarized in Table I. Although Li does not prefer to cluster in cis-PA and can store up to $10.8 \mathrm{wt} \%$ hydrogen, it binds weakly to hydrogen and can only be used for storing hydrogen at low temperatures. On the other hand, $\mathrm{B}$ prefers to cluster in cis-PA as Ti does. While B-doped cis-PA can bind up to 7.5 wt \% hydrogen, it binds to hydrogen strongly and may not be suitable for applications at ambient thermodynamic conditions. Ti-decorated cis-PA offers a compromise. The Ti-H binding energy of $0.96 \mathrm{eV}$ is ideal for hydrogen storage application, even though the gravimetric density of Ti-doped cis-PA can only reach as high as 5.1 wt $\%$.

This work is supported in part by the National Science Foundation (NSF). The research described in this Brief Report was performed in the Environmental Molecular Sciences Laboratory, a national scientific user facility sponsored by the Office of Biological and Environmental Research, Department of Energy located at Pacific Northwest National Laboratory.
${ }^{1}$ P. Chen, Z. T. Xiong, J. Z. Luo, J. Y. Lin, and K. L. Tan, Nature (London) 420, 302 (2002).

${ }^{2}$ G. G. Tibbetts, G. P. Meisner, and C. H. Olk, Carbon 39, 2291 (2001)

${ }^{3}$ H. Kajiura, S. Tsutsui, K. Kadono, M. Kakuta, M. Ata, and Y. Murakami, Appl. Phys. Lett. 82, 1105 (2003).

${ }^{4}$ M. Shiraishi, T. Takenobu, and M. Ata, Chem. Phys. Lett. 367, 633 (2003).

${ }^{5}$ R. E. Barajas-Barraza and R. A. Guirado-Lopez, Phys. Rev. B 66, 155426 (2002).

${ }^{6}$ N. L. Rosi, J. Eckert, M. Eddaoudi, D. T. Vodak, J. Kim, M. O'Keeffe, and O. M. Yaghi, Science 300, 1127 (2003).

${ }^{7}$ X. Blase, A. Rubio, S. G. Louie, and M. L. Cohen, Europhys. Lett. 28, 335 (1994).

${ }^{8}$ Q. Y. Wang and J. K. Johnson, J. Chem. Phys. 110, 577 (1999).

${ }^{9}$ Y. F. Zhao, Y. H. Kim, A. C. Dillon, M. J. Heben, and S. B. Zhang, Phys. Rev. Lett. 94, 155504 (2005).

${ }^{10}$ T. Yildirim and S. Ciraci, Phys. Rev. Lett. 94, 175501 (2005).

${ }^{11}$ Q. Sun, P. Jena, Q. Wang, and M. Marquez, J. Am. Chem. Soc. 128, 9741 (2006).

${ }^{12}$ O. Sun, Q. Wang, P. Jena, and Y. Kawazoe, J. Am. Chem. Soc.
127, 14582 (2005).

${ }^{13}$ H. Lee, W. I. Choi, and J. Ihm, Phys. Rev. Lett. 97, 056104 (2006).

${ }^{14}$ E. Durgun, S. Ciraci, W. Zhou, and T. Yildirim, Phys. Rev. Lett. 97, 226102 (2006).

${ }^{15}$ S. Li and P. Jena, Phys. Rev. Lett. 97, 209601 (2006).

${ }^{16}$ I. Cabria, M. J. Lopez, and J. A. Alonso, J. Chem. Phys. 123, 204721 (2005).

${ }^{17}$ A. Udomvech, T. Kerdcharoen, and T. Osotchan, Chem. Phys. Lett. 406, 161 (2005).

${ }^{18}$ J. H. Cho and C. R. Park, Catal. Today 120, 407 (2007).

${ }^{19}$ J. P. Perdew, K. Burke, and M. Ernzerhof, Phys. Rev. Lett. 77, 3865 (1996).

${ }^{20}$ W. Kohn and L. J. Sham, Phys. Rev. 140, A1133 (1965).

${ }^{21}$ P. E. Blöchl, Phys. Rev. B 50, 17953 (1994).

${ }^{22}$ G. Kresse and J. Furthmüller, Phys. Rev. B 54, 11169 (1996).

${ }^{23}$ A. Ramirez-Solis, C. M. Zicovich-Wilson, and B. Kirtman, J. Chem. Phys. 124, 244703 (2006).

${ }^{24}$ B. K. Rao and P. Jena, Europhys. Lett. 20, 307 (1992).

${ }^{25}$ Z. Zhou, X. P. Gao, J. Yan, and D. Y. Song, Carbon 44, 939 (2006). 